\title{
Estudio bibliométrico de las primeras cinco revistas de comunicación indexadas en Web of Science 2021
}

\section{Bibliometric study of the five communication journals indexed in Web of Science 2021}

José Luis Vázquez Luna*

Universidad Anáhuac México, Facultad de Comunicación

jvazquez@anahuac.mx

https://orcid.org/0000-0002-8808-5023

Av. Universidad Anáhuac núm. 46, col. Lomas Anáhuac,

Huixquilucan, Estado de México, C.P. 52786, México

Editor: Rogelio del Prado Flores

Fecha de recepción: 26 de octubre de 2021

Fecha de aceptación: 2 de diciembre de 2021

https://doi.org/10.36105/stx.2022n8.04

\section{RESUMEN}

Se realiza un estudio bibliométrico de las cinco revistas de comunicación indexadas en el primer cuartil en la base de datos Web of Science, se presentan sus datos generales y se desglosa su factor de impacto y el número de citas; también se muestran los principales países que aportan conocimientos al área de comunicación en las revistas analizadas. Por otra parte se presenta el índice de inmediatez de los artículos publicados, que es la posibilidad de que un artículo sea citado por otro investigador el mismo año de su publicación. Como resultados encontramos que los principales países que aportan documentos a estas revistas son: Estados Unidos de América, Inglaterra, China, Alemania y Canadá, entre otros; también se observó que las cinco revistas tienen un índice de inmediatez cercano a uno.

Palabras clave: comunicación, indexación, bases de datos científicas, evaluación, investigación.

\footnotetext{
* Doctor en Comunicación Aplicada por la Universidad Anáhuac México. Maestro en Administración de Negocios por el Instituto Politécnico Nacional (IPN), licenciado en Biblioteconomía por la Escuela Nacional de Biblioteconomía y Archivonomía. Profesor de posgrado en la Universidad Anáhuac México.
} 


\section{ABSTRACT}

In this document, a bibliometric study of the five Communication journals indexed in the first quartile in the Web of Science database is carried out, their general data is presented and their impact factor and the number of citations are broken down, the main countries that contribute knowledge to the area of communication in the analyzed journals, on the other hand, the immediacy index of the published articles is presented, the immediacy index is the possibility that an article is cited by another researcher the same year of its publication.. As results we find that the main countries that contribute documents to these journals are: United States of America, England, China, Germany and Canada, among others, it can also be seen that the five journals have an index immediacy close to one.

Keywords: communication, indexing, scientific databases, evaluation, investigation.

\section{INTRODUCCIÓN}

Desde hace muchos años el conocimiento científico ha sido considerado como un instrumento que permite entender la situación que vive la sociedad, a través de la investigación y la ciencia el ser humano tiene mayor posibilidad de transformar el entorno en todos los sentidos, de ahí que se ha llegado a considerar como un capital social (Eslava, 20I8), y es a través de la publicación de los trabajos realizados por los investigadores que se socializa el conocimiento y el público se entera de los avances y las propuestas que hacen estudiar los fenómenos que impactan en el ecosistema.

El uso y la generación de información no es algo estático, ha ido cambiando de acuerdo con las necesidades y circunstancias que se van presentando en la realidad, esto lo podemos constatar con la evolución que ha tenido el concepto desarrollado por Manuel Castells (1996), sociedad de la información, que hace referencia a la generación, el procesamiento y la transmisión de información que se convierten en las fuentes fundamentales de la productividad, es decir, una relación pasiva. Ahora, en la sociedad del conocimiento se habla de un proceso cognitivo que requiere una capacidad suficiente para interpretar y manipular los datos y generar un nuevo conocimiento.

Algo interesante que sucedió en 2020 durante la pandemia, fue que a nivel nacional se diera un crecimiento en el uso de lo digital y aunque varios sectores productivos ya trabajaban en su implementación, a decir de los expertos, esta situación permitió avanzar entre 6 y io años en el uso de la tecnología, esto no fue opcional, la sociedad se vio obligada a alfabetizarse en términos digitales para ser más productivos. 
Dentro de todo este cambio y adaptación de la realidad, la demanda por parte de la sociedad hacia las universidades en su parte de investigación ha sido más fuerte para que presenten trabajos que ayuden a solucionar los problemas de la sociedad.

\section{Planteamiento del problema}

En el documento Latinoamérica: producción científica y tendencias de crecimiento (Tibaná, 202I), se establece que a pesar de la disminución en la producción científica de Argentina, Venezuela, Cuba y Puerto Rico, en comparación con hace cinco años, países como México ha incrementado su producción llegando prácticamente al mismo nivel de Brasil, que es el país con mayor producción en la región. También se establece que Perú, Colombia, Ecuador y Honduras han tenido un aumento considerable logrando niveles muy destacables. Específicamente en el caso de Ecuador y Perú se pronostica que, de mantener su ritmo, pronto estarían muy cerca del nivel de México.

Ante este aumento en la producción científica, los investigadores se ven obligados a buscar una revista para publicar su trabajo y dar a conocer sus avances o resultados, para esto existen infinidad de revistas en todos los países, sin embargo, al investigador le interesa ser publicado en revistas con un alto reconocimiento, por lo que se debe enfocar en los principales índices que hay en el mercado (Scopus y Web fo Science). Pero ya estando ahí, cómo identificar aquellas publicaciones en la que pueda enviar su trabajo.

En este documento se analizarán las revistas indexadas en Web of Science en el área específica de comunicación, haciendo una clasificación por cuartil, por factor de impacto y explicando algunos de los principales indicadores bibliométricos que presenta esta base de datos.

\section{METODOLOGÍA}

Se consultó la base de datos Journal Citation Report, dentro de Web of Science, y se realizó una búsqueda en el apartado de examinar categorías. Cabe destacar que dentro de este rubro se integran 254 áreas o disciplinas en las que se agrupa el conocimiento humano. El siguiente paso fue seleccionar la categoría Ciencias sociales, general, en la que se insertan 4I disciplinas, de las cuales se eligió Comunicación, en la base de Social Science Citation Index, que incluye 94 revistas. El siguiente paso fue obtener el resultado de esta búsqueda y presentar sus datos de identificación; posteriormente, se agruparon y analizaron las primeras cinco revistas que encabezan la lista y están ubicadas en el cuartil I, de ellas se examinó el factor de impacto, el número de citas, los países que aportan conocimiento a este campo, las instituciones que participan en la producción científica y el índice de inmediatez. 


\section{ABORDAJE TEÓRICO}

Las ciencias sociales no se distinguen por el uso de las matemáticas como herramienta principal, sin embargo, ha sido necesario incorporarlas para ayudar a explicar y complementar las investigaciones realizadas en esta área del conocimiento humano. La incorporación de los números se dio principalmente con el positivismo de Augusto Comte, posteriormente con el desarrollo de disciplinas como la bibliometría, cienciometría y la infometría, en los años sesenta, derivadas de la biblioteconomía y la documentación (Chaviano, 2004).

Este documento se desarrolla tomado los principios básicos de la bibliometría que tiene como objetivo fundamental la aplicación de las matemáticas y estudios estadísticos para estudiar el comportamiento de una disciplina científica en particular. Para llevar a cabo el análisis de los procesos y comportamiento de la información, se utilizan una serie de indicadores que ayudan a medir la calidad e importancia de las publicaciones, el impacto que tienen las revistas en la comunidad científica, entre otros. Con estos indicadores se puede determinar: cómo se ha comportado un campo científico a través del tiempo, determinar la vida "útil" de los documentos de acuerdo con el área que pertenecen, conocer la productividad científica de un investigador, todos estos indicadores son importantes pues al conocer el rumbo y comportamiento de un área en particular, ayudan a las personas responsables a tomar mejores decisiones (Ardanuy, 20I2).

\section{DESARROLLO}

El primer elemento considerado en este documento son los datos de identificación de las revistas, (Tabla I), de los cuales se puede destacar que de las cinco revistas analizadas, tres son de Inglaterra y dos de Estados Unidos de América, asimismo, dos proveedores tienen dos revistas cada una; también se puede observar que las dos primeras revistas se enfocan únicamente al tema de comunicación, las otras tres cubren otras áreas como ciencias políticas, negocios, sociología y ciencias sociales, es decir, contienen información de otras áreas además de la comunicación; el orden presentado está dado por el número total de las citas acumuladas por cada revista.

El siguiente elemento considerado fue el factor de impacto, el cual es uno de los principales indicadores para medir la importancia relativa de una revista dentro de un campo científico, no es válido tratar de hacer un comparativo con revistas pertenecientes a campos diferentes pues su utilización y cobertura son diferentes. 
62 ESTUDIO BIBLIOMÉTRICO DE LAS PRIMERAS CINCO REVISTAS DE COMUNICACIÓN INDEXADAS - JOSÉ LUIS VÁZOUEZ LUNA

TABLA 1. DATOS DE IDENTIFICACIÓN DE LAS REVISTAS

\begin{tabular}{|l|l|l|l|l|l|c|}
\hline \multicolumn{1}{|c|}{ NOMBRE DE LA REVISTA } & \multicolumn{1}{|c|}{ ISSN } & EISSN & REGIÓN & PROVEEDOR & CATEGORÍA & $\begin{array}{c}\text { TOTAL } \\
\text { CITATIONS }\end{array}$ \\
\hline $\begin{array}{l}\text { Journal of } \\
\text { Communication }\end{array}$ & $0021-9916$ & $1460-2466$ & USA & Oxford Univ. & Comunicación & 11,437 \\
\hline New Media \& Society & $1461-4448$ & $1461-7315$ & England & Sage Publicat & Comunicación & 10,325 \\
\hline $\begin{array}{l}\text { Public Opinion } \\
\text { Quarterly }\end{array}$ & $0033-362 X$ & $1537-5331$ & England & Oxford Univ. & $\begin{array}{l}\text { Ciencia política, } \\
\text { Comunicación, } \\
\text { Ciencias Sociales }\end{array}$ & 7,999 \\
\hline $\begin{array}{l}\text { Journal of Advertising } \\
\text { Ong }\end{array}$ & $0091-3367$ & $1557-7805$ & USA & $\begin{array}{l}\text { Routledge } \\
\text { Journals } \\
\text { Taylor \& }\end{array}$ & $\begin{array}{l}\text { Comunicación } \\
\text { y negocios }\end{array}$ & 6,977 \\
\hline $\begin{array}{l}\text { Information } \\
\text { Communication \& } \\
\text { Society }\end{array}$ & $1369-118 X$ & $1468-4462$ & England & $\begin{array}{l}\text { Routledge } \\
\text { Journals } \\
\text { Taylor \& }\end{array}$ & $\begin{array}{l}\text { Comunicación } \\
\text { y Sociología }\end{array}$ & 6,684 \\
\hline
\end{tabular}

FUENTE: ELABORACIÓN PROPIA CON DATOS DE WEB OF SCIENCE, 2021.

Para obtener el factor de impacto de una revista en particular en un año (2020), se considera la suma del número de citas recibidas en el año que se quiere conocer (2019) y el año previo (20I8), este dividido entre el número de artículos publicados en el mismo periodo de tiempo (2018-20I9) (Figura I).

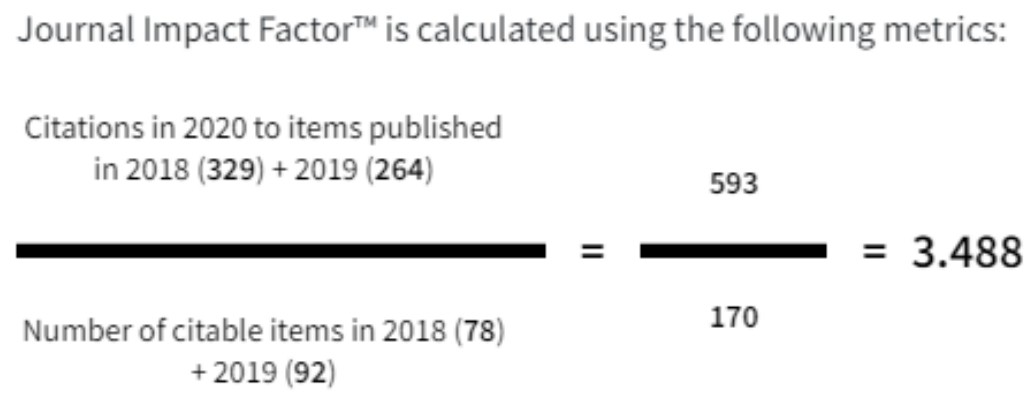

FIGURA 1. FÓRMULA PARA OBTENER EL FACTOR DE IMPACTO DE UNA REVISTA

FUENTE: JOURNAL CITATION REPORT, 2021. 
Respecto al factor de impacto se puede observar que solo dos revistas han recibido en total más de I,000 citas, otras dos tienen menos de 500 y solo una tiene más de 600 , esto contrasta con el número de artículos citables, donde tres revistas tienen menos de roo. Cabe destacar que la diferencia entre el primer lugar y los segundos, es de tres puntos (Tabla 2).

TABLA 2. FACTOR DE IMPACTO 2020

\begin{tabular}{|c|c|c|c|c|c|c|c|}
\hline \multirow[b]{2}{*}{ NOMBRE DE LA REVISTA } & \multicolumn{2}{|c|}{$\begin{array}{l}\text { CITAS EN } 2020 \\
\text { PARA ITEMS } \\
\text { PUBLICADOS }\end{array}$} & \multirow[t]{2}{*}{ TOTAL } & \multicolumn{2}{|c|}{$\begin{array}{l}\text { NÚMERO DE } \\
\text { ITEMS CITABLES }\end{array}$} & \multirow[t]{2}{*}{ TOTAL } & \multirow[t]{2}{*}{$\begin{array}{l}\text { FACTOR DE } \\
\text { IMPACTO }\end{array}$} \\
\hline & 2018 & 2019 & & 2018 & 2019 & & \\
\hline New Media \& Society & 2,312 & 735 & 3,047 & 245 & 133 & 378 & 8.061 \\
\hline Journal of Communication & 524 & 123 & 647 & 59 & 30 & 89 & 7.27 \\
\hline Journal of Advertising & 211 & 159 & 370 & 26 & 41 & 67 & 5.522 \\
\hline Information Communication \& Society & 685 & 562 & 1,247 & 114 & 116 & 230 & 5.422 \\
\hline Public Opinion Quarterly & 211 & 113 & 324 & 40 & 38 & 78 & 4.154 \\
\hline
\end{tabular}

FUENTE: ELABORACIÓN PROPIA CON DATOS DE WEB OF SCIENCE, 2021.

Una de las características que identifica a la base de datos Web of Science es su alcance a nivel internacional y la cantidad de artículos incorporados en las revistas indexadas. Ante esta situación podemos observar que la revista en la que menos países han participado tiene I 8 y la de mayor diversidad cuenta con 48 (Tabla 3), los países que se encuentran en todas ellas son Estados Unidos de América, Inglaterra, Alemania, China, y Canadá (Tabla 4). 
TABLA 3. PAÍSES PARTICIPANTES EN LAS PRIMERAS CINCO REVISTAS INDEXADAS EN WEB OF SCIENCE

\begin{tabular}{|l|c|}
\hline \multicolumn{1}{|c|}{ NOMBRE DE LA REVISTA } & PAÍSES PARTICIPANTES \\
\hline New Media \& Society & 48 \\
\hline Journal of Communication & 27 \\
\hline Journal of Advertising & 25 \\
\hline Information Communication \& Society & 54 \\
\hline Public Opinion Quarterly & 18 \\
\hline
\end{tabular}

FUENTE: ELABORACIÓN PROPIA CON DATOS DE WEB OF SCIENCE, 2021.

En las revistas Public Opinion Quarterly, Fournal of Communication y New Media \& Society aparece México como contribuyente con un artículo en cada una de ellas.

Por otra parte, otro elemento que se analizó fue el índice de inmediatez de las revistas, este indicador nos ayuda a conocer la posibilidad de que un artículo sea citado por otro investigador el mismo año en que fue publicado en la revista. Se calcula dividiendo el número de citas recibidas por los artículos entre el número de artículos publicados (Figura 2).

\section{Immediacy Index calculation}

Cites in 2020 to items published in 2020

Number of items published in 2020

$$
299 / 202=1.480
$$

FIGURA 2. FÓRMULA PARA CALCULAR EL ÍNDICE DE INMEDIATEZ

FUENTE: JOURNAL CITATION REPORT, 2021. 
TABLA 4. PAÍSES CONTRIBUYENTES Y NÚMERO DE ARTÍCULOS QUE APORTAN

\begin{tabular}{|l|c|l|c|l|c|}
\hline \multicolumn{2}{|c|}{$\begin{array}{c}\text { INFORMATION } \\
\text { COM MUNICATION \& SOCIETY }\end{array}$} & \multicolumn{2}{c|}{ JOURNAL OF ADVERTISING } & \multicolumn{2}{c|}{ PUBLIC OPINION QUARTERLY } \\
\hline PAís CONTRIBUYENTE & ARTÍCULOS & PAÍS CONTRIBUYENTE & ARTíCULOS & PAÍS CONTRIBUYENTE & ARTí́cULOS \\
\hline USA & 150 & USA & 72 & USA & 106 \\
\hline England & 123 & England & 13 & England & 9 \\
\hline Australia & 44 & Netherlands & 12 & Germany (FED REP GER) & 8 \\
\hline Canada & 42 & China Mainland & 10 & Canada & 5 \\
\hline $\begin{array}{l}\text { Germany } \\
\text { (FED REP GER) }\end{array}$ & 30 & Germany & 9 & Israel & 3 \\
\hline Netherlands & 30 & South Corea & 8 & Netherlands & 3 \\
\hline Israel & 24 & Canada & 6 & Scotland & 2 \\
\hline China Mainland & 20 & Australia & 5 & Switzerland & 2 \\
\hline Norway & 18 & Belgium & 5 & Australia & 1 \\
\hline Italy & 16 & New Zeland & 5 & Austria & 1 \\
\hline
\end{tabular}

\begin{tabular}{|l|c|l|c|}
\hline \multicolumn{2}{|c|}{ JOURNAL OF COMMUNICATION } & \multicolumn{2}{c|}{ NEW MEDIA \& SOCIETY } \\
\hline PAís CONTRIBUYENTE & ARTícULOS & \multicolumn{1}{c|}{ PAÍs CONTRIBUYENTE } & ARTí́CULOS \\
\hline USA & 115 & USA & 319 \\
\hline England & 13 & England & 112 \\
\hline China Mainland & 11 & Australia & 56 \\
\hline Netherlands & 11 & Germany (FED REP GER) & 49 \\
\hline Canada & 10 & Netherlands & 47 \\
\hline Germany (FED REP GER) & 9 & Canada & 44 \\
\hline Sngapore & 6 & China Mainland & 30 \\
\hline Australia & 4 & Israel & 30 \\
\hline Austria & 4 & Sweden & 27 \\
\hline Denmark & 4 & Finland & 22 \\
\hline
\end{tabular}

FUENTE: ELABORACIÓN PROPIA CON DATOS DE WEB OF SCIENCE, 2021. 
En la Tabla 5 se puede observar que los artículos de la revista Information Communication \& Society tienen la más alta posibilidad de ser citados el mismo año de su publicación con I.48, y los artículos de las cuatro restantes están muy cerca del uno, lo cual indica una buena posibilidad para que sean citados.

TABLA 5. FACTOR DE INMEDIATEZ DE LAS REVISTAS

\begin{tabular}{|l|c|}
\hline \multicolumn{1}{|c|}{ NOMBRE DE LA REVISTA } & INMEDIATEZ \\
\hline New Media \& Society & 0.963 \\
\hline Journal of Communication & 0.974 \\
\hline Journal of Advertising & 0.959 \\
\hline Information Communication \& Society & 1.48 \\
\hline Public Opinion Quarterly & 0.882 \\
\hline
\end{tabular}

FUENTE: ELABORACIÓN PROPIA CON DATOS DE WEB OF SCIENCE, 2021.

\section{CONCLUSIONES}

En el análisis realizado a las cinco primeras revistas del área de comunicación indexadas en el Web of Science podemos darnos cuenta de la importancia que tienen los estudios bibliométricos, pues a través de ellos los investigadores pueden identificar a qué revista deben enfocar sus esfuerzos para publicar y también en cuáles pueden encontrar los trabajos con mayor impacto en el medio en que se desarrollan.

Es cierto que las revistas publicadas e indexadas en bases de datos regionales ayudan a promover el desarrollo de las áreas de conocimiento y dar a conocer los trabajos de los investigadores, lo hacen de forma local y el impacto que tienen es más limitado, al publicar en revistas indexadas en repositorios internacionales, el alcance será mayor y los trabajos tendrán un mayor impacto. 


\section{REFERENCIAS}

Ardanuy, J. (2012). Breve introducción a la bibliometría. Universitat de Barcelona. http://diposit. ub.edu/dspace/bitstream/2445/30962/r/breve\%2ointroduccion\%2obibliometria.pdf

Castells, M. (1996). La era de la información. Economía, sociedad y cultura, vol. I. Siglo XXI.

Chaviano, O. G. (2004). Algunas consideraciones teórico-conceptuales sobre las disciplinas métricas. Acimed, I2(5), pp. I-I. https://core.ac.uk/download/pdf/2904700I5.pdf

Eslava, D. G. (20I8). La función social de la investigación. Investigaciones Andina, 2o(36), pp. 5-8. https://www.redalyc.org/journal/2390/23905978800I/html/

Tibaná, G. (202I, 20 de abril). Latinoamérica: producción científica y tendencias de crecimiento. SCImago Lab. https://www.scimagolab.com/latinoamerica-produccion-cientifica-y-tendencias-de-crecimiento/

Esta obra está bajo Licencia Creative Commons Atribución-NoComercial-SinDerivadas 4.0 Internacional. 